\title{
Early post-operative cerebrospinal fluid hypovolemia: Report of 7 cases
}

\author{
$\mathrm{KUN} \mathrm{HOU}^{1 *}, \mathrm{XIAOBO} \mathrm{ZHU}^{1 *}$, YANG ZHANG $^{1}, \mathrm{XIANFENG} \mathrm{GAO}^{1}$, \\ SHIHUAN SUO ${ }^{1}$, JINCHUAN ZHAO ${ }^{1}$ and GUICHEN LI $^{2}$
}

Departments of ${ }^{1}$ Neurosurgery and ${ }^{2}$ Neurology, The First Hospital of Jilin University,

Changchun, Jilin 130021, P.R. China

Received October 4, 2017; Accepted January 9, 2018

DOI: $10.3892 /$ etm.2018.6018

\begin{abstract}
Cerebrospinal fluid (CSF) hypovolemia is a common neurosurgical condition, which may be spontaneous or iatrogenic. At our institution, a substantial number of the reported cases of early post-operative CSF hypovolemia were identified to have unintentional or unrecognized post-operative continuous excessive CSF leakage. Cases who presented with post-operative CSF hypovolemia several days after uneventful intracranial surgeries without continuous CSF leakage were rarely reported. A retrospective review of the medical records of these patients was performed to identify those patients who developed early post-operative CSF hypovolemia without the presence of post-operative continuous CSF leakage. A total of 7 patients, 5 of which were males, were identified in this retrospective study. They experienced CSF hypovolemia between days 1 and 7 after emergency or scheduled intracranial surgeries. Ventricular collapse, cisternal effacement and midline shift are the most common radiological observations. With early diagnosis and management, 4 of the patients achieved a Glasgow Outcome Scale (GOS) score of 5, 1 achieved a GOS score of 4 and the remaining 2 had a GOS score of 3. No mortality was noted in this series. Although rare in incidence, early post-operative CSF hypovolemia may occur without the
\end{abstract}

Correspondence to: Dr Guichen Li, Department of Neurology, The First Hospital of Jilin University, 71 Xinmin Avenue, Changchun, Jilin 130021, P.R. China

E-mail: gretchen666@yeah.net

Dr Jinchuan Zhao, Department of Neurosurgery, The First Hospital of Jilin University, 71 Xinmin Avenue, Changchun, Jilin 130021, P.R. China Email: hanzhiguoscience@163.com

\section{${ }^{*}$ Contributed equally}

Abbreviations: CSF, cerebrospinal fluid; ICP, intracranial pressure; SDE, subdural effusion; GOS, Glasgow Outcome Scale; CT, computed tomography; GCS, Glasgow Coma Scale; IH, intracranial hypotension

Key words: cerebrospinal fluid hypovolemia, intracranial hypotension, cerebrospinal fluid leakage, early postoperative existence of post-operative continuous CSF leakage. When the diagnosis of CSF hypovolemia is reached, factors that may exacerbate CSF compensation should be promptly terminated. Trendelenburg position and sufficient intravenous hydration are practical and effective managements, and CSF hypovolemia may thereby be reversed in a substantial number of patients.

\section{Introduction}

Cerebrospinal fluid (CSF) hypovolemia, also referred to as intracranial hypotension ( $\mathrm{IH})$, is a common neurosurgical scenario, which may occur spontaneously or be iatrogenic. The clinical manifestation ranges from headache, cranial nerve palsy to mental state decline, and may result in death (1-4). Based on the medical history and characteristic symptoms of affected patients the diagnosis is relatively straight-forward. Regardless of the causes, patients with CSF hypovolemia frequently have pre-existent continuous CSF leakage (1-5). Post-operative CSF hypovolemia is a specific type of CSF hypovolemia and a substantial number of the reported cases of early post-operative CSF hypovolemia were identified to had unintentional or unrecognized post-operative continuous excessive CSF leakage according to further investigation (1-3,6-9). The most severe types of post-operative CSF hypovolemia are pseudohypoxic brain swelling (PHBS) and post-operative $\mathrm{IH}$-associated venous congestion (PIHV), which is characterized by effacement of basal cisterns, ventricular collapse, venous congestion and changes in bilateral deep gray structures on imaging $(3,6-9)$. As a result of the excessive loss of CSF through lumbar drainage or sub-galeal suction drainage over a short period, patients with PHBS or PIHV often experience irreversible progressive exacerbation and death. The present study provided another series of patients who were diagnosed with post-operative CSF hypovolemia after uneventful intracranial surgery. Of note, in contrast to previous studies, no post-operative lumbar or sub-galeal suction drainage was placed. Due to early recognition and timely management, the affected patients experienced relatively favorable outcomes.

\section{Case report}

Between January 2011 and December 2015, 2,748 emergencies or scheduled intracranial surgeries were performed at 
the Department of Neurosurgery of the First Hospital of Jilin University (Eastern Division; Changchun, China). A retrospective review of the medical records of the patients was performed to identify those who developed early post-operative CSF hypovolemia without the existence of continuous CSF leakage. The present study was approved by the Ethics Committee of The First Hospital of Jilin University (Changchun, China). Written informed consent for the publication of their data and any accompanying images was obtained from the patients or their guardians. The definition of early post-operative CSF hypovolemia without continuous CSF leakage was as follows: i) Progressive decline in mental state or other neurological symptoms that were not explainable by factors that may cause an acute increase in intracranial pressure (e.g., intracranial bleeding, massive ischemic stroke and hydrocephalus); ii) drainage modalities, as lumbar drainage and sub-galeal suction drainage were absent; iii) evidence of CSF hypovolemia by computed tomography (CT) (e.g., collapse of the ventricular system, effacement of the cisterns, sub-dural collection, brain sag and intracranial venous engorgement); iv) Low or normal intracranial pressure (ICP) identified by ICP monitoring or lumbar puncture; v) Exclusion of intra- and post-operative cerebral hypoxia; vi) evident improvement of the clinical symptoms when management for CSF hypovolemia was initiated.

Patient characteristics. A total of 7 patients, 5 of which were male, were identified in the present retrospective review (Table I). The patient age ranged from 44 to 61 years (mean, 47.86 years). They experienced CSF hypovolemia between days 1 and 7 after emergency or scheduled intracranial surgery. Ventricular collapse, cisternal effacement and midline shift are the most common radiological observations. Sub-dural effusion (SDE) was identified in two patients, one of whom experienced spontaneous absorption after mannitol ceasing and intravenous hydration, and the other one underwent decompressive craniectomy to halt irreversible progressive decline in mental state. A total of 4 patients achieved a Glasgow Outcome Scale (GOS) score of 5, 1 achieved a GOS of 4 and the other 2 a GOS of 3 . No mortality was noted in this series.

\section{Illustrative cases}

Case 1. A 47-year-old healthy man was admitted after a car accident. Head CT revealed an acute subdural hematoma at the right frontoparietotemporal region and an evident midline shift to the left side (Fig. 1A). An emergent decompressive craniectomy was performed. A head CT performed at 1 day post-operatively revealed complete evacuation of the subdural hematoma and restoration of the midline shift (Fig. 1B). The patient experienced an uneventful recovery process until his consciousness began to decline on post-operative day 5. Although the decompressive site was soft on palpation and the ICP monitoring result was $80 \mathrm{~mm}$ $\mathrm{H}_{2} \mathrm{O}$, CT revealed brain swelling, collapse of the ventricular system, effacement of the cisterns and midline shift to the left side (Fig. 1C and D). Intravenous (iv) administration of $50 \mathrm{~g}$ mannitol three times a day (tid) was prescribed. The patient's mental state continued to decline. CT performed at 10 days post-operatively revealed exacerbation of the brain swelling and effacement of the cisterns, and a more evident midline shift. The presence of CSF hypovolemia was then considered.
Intravenous mannitol was ceased and intravenous hydration and Trendelenburg position were subsequently initiated, following which the patient's consciousness was obviously regained. CT performed at 14 days post-operatively indicated re-expansion of the ventricular system and cisterns, as well as restoration of the midline (Fig. 1E and F). The patient experienced a favorable recovery and a custom-made titanium cranioplasty was performed after 4 months. His GOS score was 5 at 1 year's follow-up.

Case 3. A 46-year-old woman was admitted to our department for subarachnoid hemorrhage (Fig. 2A). The subarachnoid hemorrhage was demonstrated to be rupture of a left middle cerebral aneurysm by further CT angiography. No past medical history was reported. She underwent an uneventful microsurgical clipping of the aneurysm. Administration of mannitol (50 g, tid, iv) was prescribed to prevent post-operative brain edema. Three days post-operatively, the patient's mental state declined. CT revealed basal cistern effacement and left SDE (Fig. 2B and C). The opening pressure was $110 \mathrm{~mm} \mathrm{H}_{2} \mathrm{O}$ on subsequent lumbar puncture in the lateral decubitus position. As CSF hypovolemia was suspected, mannitol was ceased and intravenous hydration was initiated, following which the patient's consciousness began to recover gradually. At 7 days post-operatively, the SDE disappeared spontaneously with midline restoration (Fig. 2D). The patient was discharged with a Glasgow Coma Scale (GCS) score of 15 (10).

Case 5. A 52-year-old man was admitted to our department following a car accident. Head CT performed at the emergency room revealed an extensive subdural hematoma at the right side (Fig. 3A). Physical examination indicated decerebrate rigidity, anisocoria and a GCS score of 4 . He had a history of diabetes for 2 years, as well as smoking and alcohol abuse for $>30$ years. An emergent decompressive craniectomy was performed. On day 1 post-operatively, he was able to withdraw from painful stimuli and his pupils were symmetrical and reactive to light. The ICP monitoring results ranged from 5 to $11 \mathrm{~mm} \mathrm{Hg}$. On day 2 post-operatively, he developed a decorticate response and anisocoria. No elevation of the ICP was noted. Immediate CT revealed epidural hematoma, collapse of the ventricular system and a midline shift to the left side (Fig. 3B). An emergency epidural hematoma evacuation was performed. However, his neurological state did not recover after the second operation. Another CT indicated no improvement of the collapse of the ventricular system and midline shift (Fig. 3C). The patient was placed in a Trendelenburg position and given sufficient intravenous hydration. His pupils began to become symmetrical and reactive. CT performed on day 5 post-operatively indicated re-expansion of the ventricular system and cisterns, as well as restoration of the midline (Fig. 3D). A custom-made titanium cranioplasty was performed 3 months later. His GOS score was 3 at 2 year's follow-up.

\section{Discussion}

According to a literature review performed as part of the present study, there are two types of post-operative CSF 


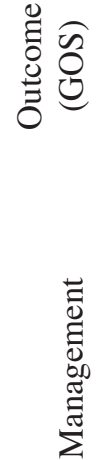

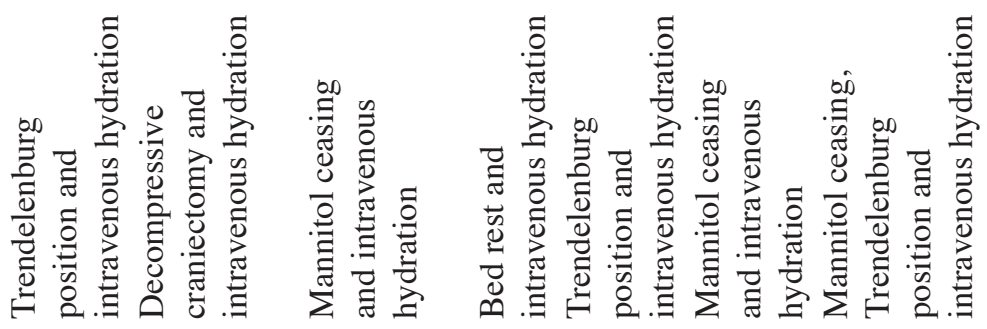

诖

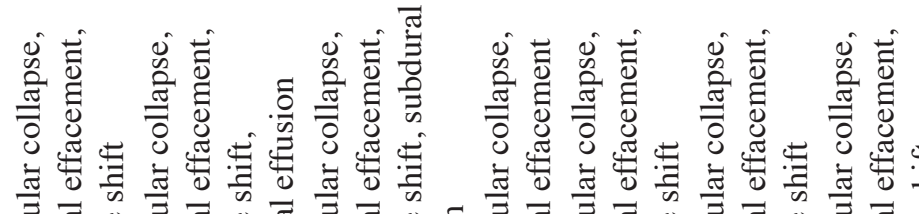

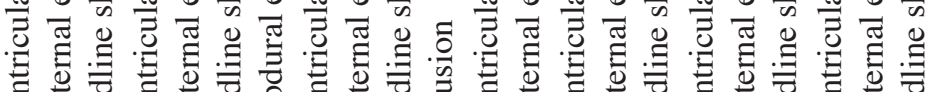

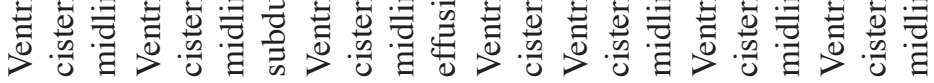

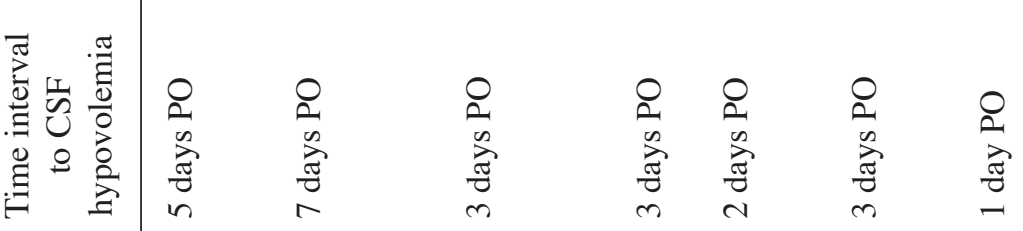

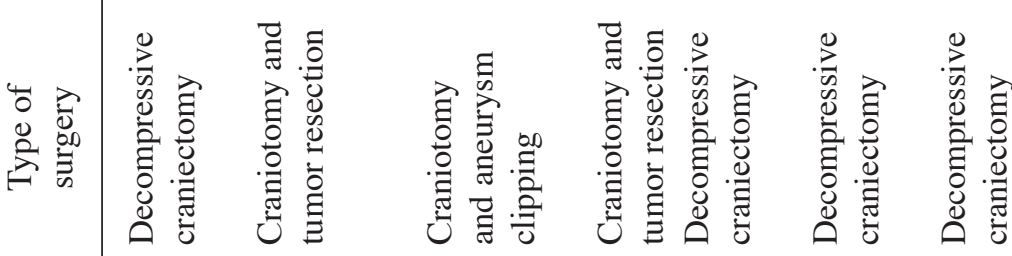

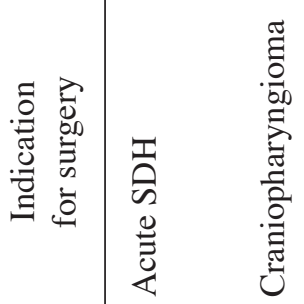

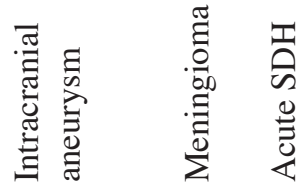

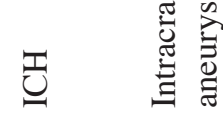

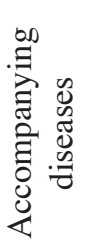

$\stackrel{0}{z}$

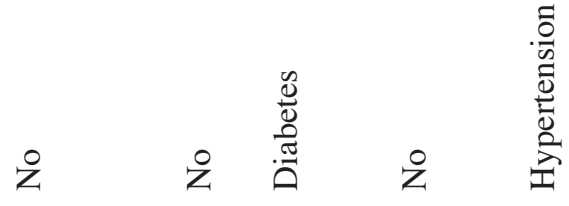

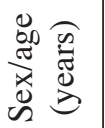

$\stackrel{\text { 守 }}{\stackrel{8}{\Sigma}}$

$\frac{\substack{1 \\ \text { I }}}{1}$

$\stackrel{v}{\Sigma} \stackrel{n}{\Sigma}$

$\stackrel{\infty}{\Sigma} \quad \stackrel{\infty}{\Sigma}$

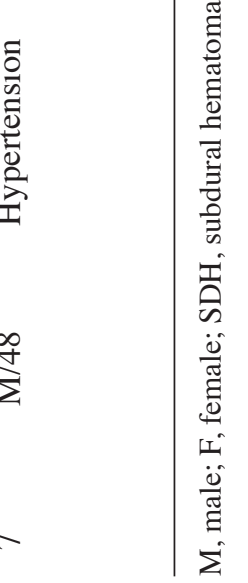


A

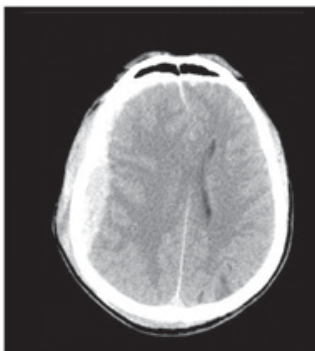

C

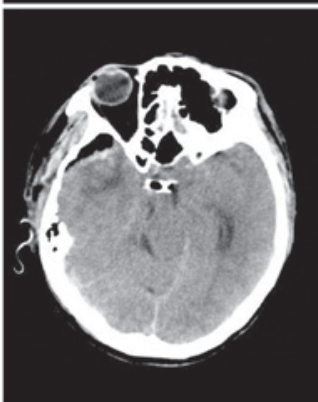

E

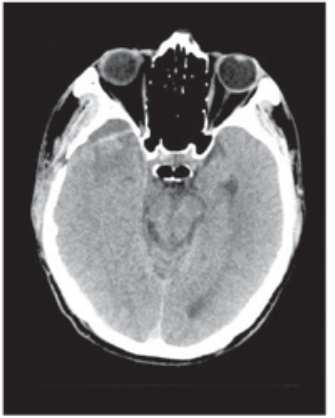

B

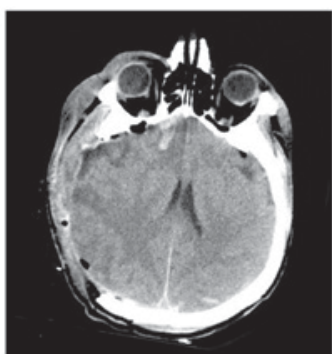

D

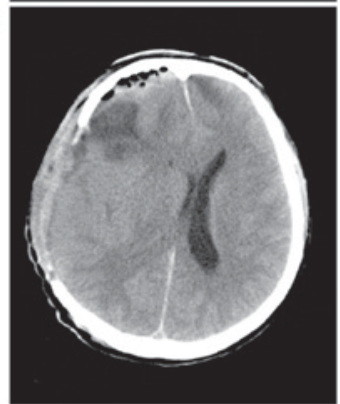

$\mathbf{F}$

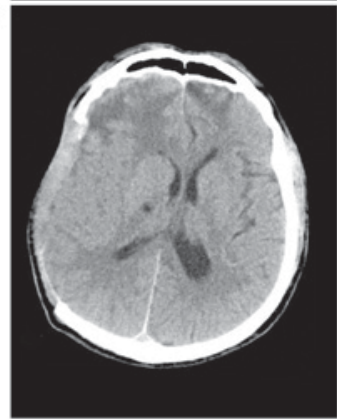

Figure 1. Head CT performed in different stages. (A) Preoperative CT indicated an acute subdural hematoma at the right frontoparietotemporal region and an evident midline shift to the left side; (B) postoperative CT indicated complete evacuation of the subdural hematoma and restoration of the midline shift; (C and D) CT on the 5th postoperative day indicated brain swelling, collapse of the ventricular system, effacement of the cisterns and midline shift to the left side; (E and F) CT performed at postoperative day 14 indicated re-expansion of the ventricular system and cisterns, and restoration of the midline. CT, computed tomography.

hypovolemia. The first one comprises an early decline in mental state or even fatal incidents due to unintentional or unrecognized continuous excessive CSF depletion (1-3,6-9). The most typical cases were reported by Van Roost et al (3), for which 17 cases of post-operative diffuse brain swelling and sulcal effacement were reported. Further investigation revealed that excessive sub-galeal suction drainage of CSF was responsible for this phenomenon. As the alterations on imaging were similar to those of cerebral hypoxia (effacement of basal cisterns, ventricular collapse, venous congestion, and changes in bilateral deep gray structures on imaging investigations), PHBS or PIHV, which are terms adopted by subsequent studies, was selected to describe this scenario to distinguish the pathology from real cerebral hypoxia (6-9). The second type features immediate deterioration in mental state after CSF depletion or sampling (usually lumbar puncture) several weeks after decompressive craniectomy (11-13). The most typical study was published recently by Creutzfeldt et al (13). Through performing a study on patients at their institution and a literature review, they concluded that lumbar puncture at $\geq 1$ month after decompressive craniectomy posed a risk

A
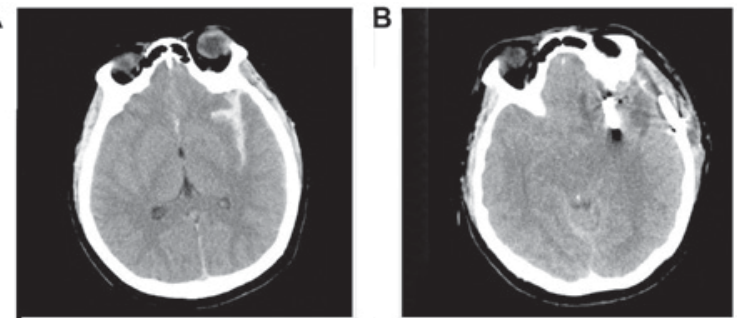

C
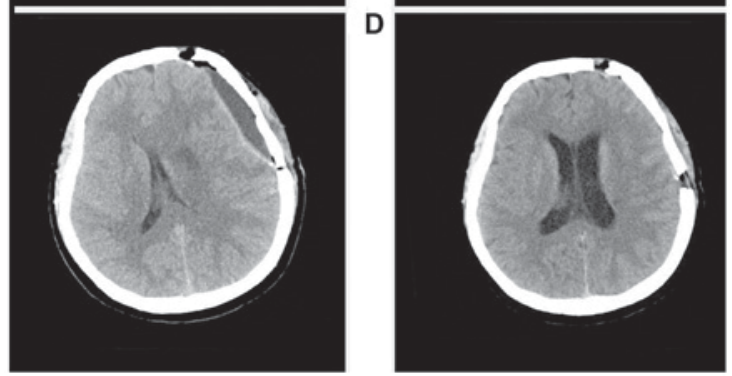

Figure 2. Head CT performed at different stages. (A) Preoperative CT indicated subarachnoid hemorrhage, (B and C) CT on postoperative day 5 indicated basal cistern effacement and left subdural effusion and (D) CT on postoperative day 7 indicated subdural effusion disappears spontaneously with midline restoration. CT, computed tomography.
A

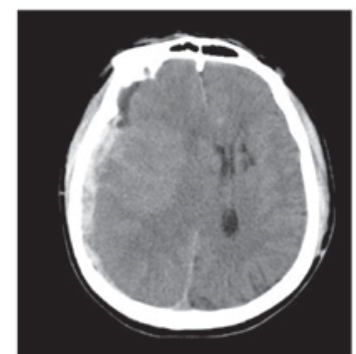

C

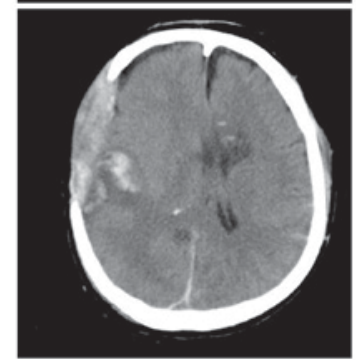

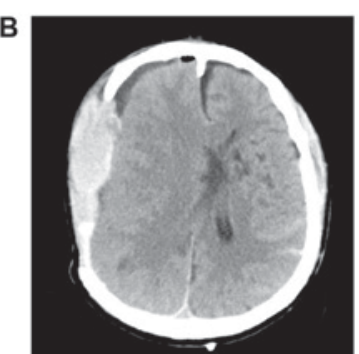

D

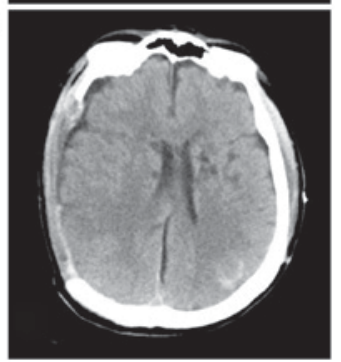

Figure 3. Head CT performed at different stages. (A) Preoperative CT indicated acute subdural hematoma at the right side, (B) CT on the 2nd postoperative day indicated epidural hematoma, collapse of the ventricular system and midline shift to the left side, (C) CT indicated collapse of the ventricular system and midline shift to the left side after the second operation, (D) CT on the 5th postoperative day indicated re-expansion of the ventricular system and cisterns, and restoration of the midline. CT, computed tomography.

of provoking paradoxical herniation. However, none of the patients developed symptomatic CSF hypovolemia in the early post-operative period of their study. Komotar et al (14) reported 11 cases of post-operative CSF hypovolemia after uneventful clipping of intracranial aneurysms in 137 patients. Although intra-operative lumbar drainage was deemed as the cause, issues regarding post-operative sub-galeal suction drainage and hyperosmotic therapy were not mentioned.

The present study provided a case series of a type of post-operative CSF hypovolemia that requires early diagnosis and management. Even though post-operative lumbar or 
sub-galeal drainage were not present, CSF hypovolemia occurred in these cases. Perhaps as a result of the absence of persistent excessive CSF depletion and early management, favorable recovery was achieved. According to our experience, the causes of CSF hypovolemia in this case series may be as follows: i) Substantial loss of CSF during surgery that was not readily compensated by post-operative CSF production, ii) inappropriate early post-operative hyperosmotic therapy exacerbated CSF depletion, iii) focal cerebral edema exacerbated the condition of CSF hypovolemia, and iv) atmospheric pressure imposed on the brain tissue due to decompressive craniectomy.

The mechanism of post-operative CSF hypovolemia was similar to that of spontaneous CSF hypovolemia. Two major pathophysiological processes prevail in this scenario. First, substantial depletion of CSF impairs its buoyancy effect on the intracranial structures. Downward migration of the intracranial contents results in traction and compression of the neurovascular structures. Subsequently, according to the doctrine of Monro-Kellie, a decrease in CSF must be compensated by other intracranial contents (15). Therefore, subdural fluid collection, engorgement of venous structures, and cytotoxic as well as vasogenic brain edema occurs, which leads to further deterioration of the mental state or even irreversible brain edema and fatal outcome $(3,4,6-9,16)$. Of note, patients with pre-existing cerebellar tonsillar herniation are more likely to have a fatal outcome $(4,17,18)$. The condition is more complex when a cranial defect is present (18). Atmospheric pressure further exacerbates compression of the neurovascular structure and downward displacement of the intracranial contents.

The terminology for describing symptoms of CSF depletion varies between different studies. Certain studies prefer the term IH, while others prefer 'CSF hypovolemia'. According to Miyazawa et al (5), certain patients with so-called IH demonstrated normal CSF pressure despite the presence of typical symptoms. The buoyancy effect of CSF on the intracranial contents depends on the CSF volume, not the intracranial pressure. The typical alterations (subdural fluid collection, engorgement of venous structures, as well as cytotoxic and vasogenic brain edema) are also due to CSF volume depletion, not intracranial pressure. According to our experience and the descriptions in other studies, we prefer the term 'CSF hypovolemia' over ' $\mathrm{IH}$ ' to describe this specific entity $(1,2,5)$.

The management of early post-operative CSF hypovolemia without continuous CSF leakage is similar to that of CSF hypovolemia caused by spontaneous or iatrogenic continuous CSF leakage. The key step is its correct and early diagnosis. When the diagnosis of CSF hypovolemia is reached, factors including continued CSF drainage, hyperosmotic agents and positions with head elevation that may exacerbate the condition should be avoided. Trendelenburg position and sufficient intravenous hydration are practical and effective methods which are able to reverse the pathology in a substantial number of patients $(1,13,18)$. In rare circumstances, an emergent decompressive sub-occipital craniectomy is lifesaving (4).

In conclusion, although rare in incidence, early post-operative CSF hypovolemia may occur without the existence of intentional or unintentional post-operative continuous CSF drainage. When the diagnosis of CSF hypovolemia is reached, factors that may impair CSF compensation should be promptly terminated. Trendelenburg position and sufficient intravenous hydration are practical and effective treatments, and the pathology was thereby reversed in a substantial number of patients.

\section{Acknowledgements}

Not applicable.

\section{Funding}

No funding was received.

\section{Availability of data and materials}

All data generated or analyzed during this study are included in this published article.

\section{Authors' contributions}

$\mathrm{KH}$ and $\mathrm{XZ}$ analyzed and interpreted the imaging data. $\mathrm{YZ}$, $\mathrm{XG}$, and SS performed a thorough review of the medical records and made substantial contributions to conception and acquisition of data. JZ and GL interpreted the clinical data and drafted the manuscript. All authors read and approved the final manuscript.

\section{Ethics approval and consent to participate}

The study was approved by the Ethics Committee at the First Hospital of Jilin University. Informed consent for participation in the study or use of their medical data was obtained from all participants or their legal guardian.

\section{Consent for publication}

Written informed consent was obtained from the patients or their guardians for publication of this manuscript and any accompanying images.

\section{Competing interests}

The authors declare that they have no competing interests.

\section{References}

1. Kawahara I, Tsutsumi K, Matsunaga Y, Takahata H, Ono T, Toda $\mathrm{K}$ and Baba H: Early awareness of cerebrospinal fluid hypovolemia after craniotomy for microsurgical aneurysmal clipping. Acta Neurochir (Wien) 155: 1543-1548, 2013.

2. Li G, Zhu X, Zhang Y, Zhao J, Han Z and Hou K: Cranial nerve palsy secondary to cerebrospinal fluid diversion. Clin Neurol Neurosurg 143: 19-26, 2016.

3. Van Roost D, Thees C, Brenke C, Oppel F, Winkler PA and Schramm J: Pseudohypoxic brain swelling: A newly defined complication after uneventful brain surgery, probably related to suction drainage. Neurosurgery 53: 1315-1326, 2003.

4. Sugrue PA, Hsieh PC, Getch CC and Batjer HH: Acute symptomatic cerebellartonsillar herniation following intraoperative lumbar drainage. J Neurosurg 110: 800-803, 2009. 
5. Miyazawa K, Shiga Y,Hasegawa T, Endoh M, Okita N, Higano S, Takahashi S and Itoyama Y: CSF hypovolemia vs intracranial hypotension in 'spontaneous intracranial hypotension syndrome'. Neurology 60: 941-947, 2003.

6. Parpaley Y, Urbach H, Kovacs A, Klehr M and Kristof RA Pseudohypoxic brain swelling (postoperative intracranial hypotension-associated venous congestion) after spinal surgery: Report of 2 cases. Neurosurgery 68: E277-E283, 2011.

7. Yokota H, Yokoyama K, Miyamoto K and Nishioka T: Pseudohypoxic brain swelling after elective clipping of an unruptured anterior communicating artery aneurysm. Clin Neurol Neurosurg 111: 900-903, 2009.

8. Evins AI, Boeris D, Burrell JC and Ducati A: Postoperative intracranial hypotension-associated venous congestion: Case report and literature review. Clin Neurol Neurosurg 115: 2243-2246, 2013.

9. Snyder KA, Clarke MJ, Gilbertson JR and Hocker SE: Prompt recognition and management of postoperative intracranial hypotension-associated venous congestion: A case report. Neurocrit Care 24: 448-453, 2016.

10. Teasdale G and Jennett B: Assessment of coma and impaired consciousness. A practical scale. Lancet 2: 81-84, 1974.

11. Oyelese AA, Steinberg GK, Huhn SL and Wijman CA: Paradoxical cerebral herniation secondary to lumbar puncture after decompressive craniectomy for a large space-occupying hemispheric stroke: Case report. Neurosurgery 57: E594, 2005.
12. Jung HJ, Kim DM and Kim SW: Paradoxical transtentorial herniation caused by lumbar puncture after decompressive craniectomy. J Korean Neurosurg Soc 51: 102-104, 2012.

13. Creutzfeldt CJ, Vilela MD and Longstreth WT Jr: Paradoxical herniation after decompressive craniectomy provoked by lumbar puncture or ventriculoperitoneal shunting. J Neurosurg 123: $1170-1175,2015$.

14. Komotar RJ, Mocco J, Ransom ER, Mack WJ, Zacharia BE, Wilson DA, Naidech AM, McKhann GM 2nd, Mayer SA, Fitzsimmons BF and Connolly ES Jr: Herniation secondary to critical postcraniotomy cerebrospinal fluid hypovolemia. Neurosurgery 57: 286-292, 2005.

15. Lundberg N: The saga of the Monro-Kellie doctrine in intracranial pressure V. In: Ishii S, Nagai H, Brock M (eds) Proceedings of the fifth international symposium on intracranial pressure. Springer, Tokyo, pp 68-76, 1983.

16. Hadizadeh DR, Kovács A, Tschampa H, Kristof R, Schramm J and Urbach $\mathrm{H}$ : Postsurgical intracranial hypotension: Diagnostic and prognostic imaging findings. AJNR Am J Neuroradiol 31: 100-105, 2010

17. Dagnew E, van Loveren HR and Tew JM Jr: Acute foramen magnum syndrome caused by an acquired Chiari malformation after lumbar drainage of cerebrospinal fluid: Report of three cases. Neurosurgery 51: 823-828, 2002.

18. Zhao J, Li G, Zhang Y, Zhu X and Hou K: Sinking skin flap syndrome and paradoxical herniation secondary to lumbar drainage. Clin Neurol Neurosurg 133: 6-10, 2015. 